\title{
SDF1/CXCR4 axis plays a role in angiogenesis during the degeneration of intervertebral discs
}

\author{
HANXIANG ZHANG and BIN HE
}

\begin{abstract}
Department of Orthopedics, The Third Affiliated Hospital of Zunyi Medical University, Zunyi, Guizhou 563000, P.R. China
\end{abstract}
Received August 30, 2018; Accepted March 15, 2019

DOI: $10.3892 / \mathrm{mmr} .2019 .10346$

\begin{abstract}
Low back pain (LBP) is a ubiquitous disease affecting quality of life. The ingrowth of new blood vessels is an important pathological feature of LBP, but its underlying mechanisms are poorly understood. The present study aimed to investigate the influence and relative mechanism of stromal cell derived factor 1 (SDF1) on the angiogenesis of degenerated intervertebral discs. The expression of SDF1 in nucleus pulposus cells (NPCs) was upregulated and downregulated by virus transfection, and the NPCs were allocated to either the downregulation (Down), degeneration (D) or upregulation (Up) group according to the expression of SDF1. The different groups of NPCs or NPC conditioned media were co-cultured with vascular endothelial cells (VECs) under different conditions. A Cell Counting Kit-8 (CCK-8) assay, a Transwell migration assay and a tube formation assay were conducted to evaluate the influence on angiogenesis. The results showed that SDF1 was significantly up- and downregulated in the Up and Down groups, respectively. Each group of NPCs or their conditioned medium was co-cultured with VECs; the CCK-8, Transwell migration and tube formation assays showed that cell viability, chemotactic migration and the tube formation ability of VECs increased with the rise in SDF1. The aforementioned results were significantly different between each group. After adding the CXCR4 inhibitor, AMD3100, the viability, migration and tube formation of VECs were suppressed in the D and Up groups, and there was a significant difference compared with the prior to the addition of the inhibitor, while there was a declining tendency in the Down group and no significant difference following addition of the inhibitor. The results demonstrated that SDF1 is expressed in human NPCs, and the SDF1/CXCR4 axis can influence the viability, migration and
\end{abstract}

Correspondence to: Dr Bin He, Department of Orthopedics, The Third Affiliated Hospital of Zunyi Medical University, 98 Fenghuang Road, Zunyi, Guizhou 563000, P.R. China E-mail: hebinyql@126.com

Key words: chemokine, C-X-C chemokine ligand 12, co-culture techniques, intervertebral disc degeneration, angiogenesis, C-X-C chemokine receptor type 4 tube formation of VECs and may play an important role in the angiogenesis of human degenerated discs.

\section{Introduction}

Low back pain (LBP) is a common condition; according to the statistics, at least $70 \%$ of people will suffer from LBP at some point in their life in western countries (1). The phenomenon of LBP is exacerbated by the aging process. In addition to the influence on life quality and self-care ability, LBP places a huge burden on the social economy (2). In 2013 alone, the cost of low back and neck pain for social healthcare in the USA was $\$ 87.6$ billion, the third highest among individual and public health costs (3). The leading cause of LBP is symptomatic intervertebral disc degeneration disease. The peripheral annulus, inner nucleus pulposus and each end of the cartilage endplate together form an intervertebral disc, and the discs create vertebral bodies in conjunction with each other and bring flexibility, cushioning and stress transmission (4). The disc is the biggest avascular organ and has a hypoxic microenvironment in its normal state (5). During degeneration, changes in the material composition of the inner disc induce changes in its mechanical properties. The loading force exceeding the endurance of the intervertebral disc leads to the formation of annulus fissures; subsequently, granulation tissue and new blood vessels grow into the fissures, simultaneously carrying inflammatory cells for infiltration (6). In adults, the extent of the intervertebral disc nerve endings does not exceed one-third of the outer annulus, and chronic LBP is often thought to be caused by a compression of the nerve endings deep in the intervertebral disc (7). Stefanakis et al (8) found that there was new vascular nerve ingrowth in annulus fissures. Freemont et al (9) presented a point of view that the blood vessels that grew into the intervertebral disc produced nerve growth factor (NGF), and the nociceptive fibers expressed a high-affinity NGF receptor that followed the growth of the blood vessels into the degenerated intervertebral disc. A study showed that neovascularization was also one of the differences between painful degenerative discs and asymptomatic degenerative intervertebral discs (10). Therefore, angiogenesis after degeneration of the intervertebral disc is important in the occurrence of LBP.

Stromal cell-derived factor 1 (SDF1), also known as C-X-C family chemokine ligand 12 (CXCL12), was initially identified as a pre-B-cell growth stimulating factor (11). CXCR4 is a seven-transmembrane-spanning $\mathrm{G}$ protein-coupled receptor 
and was identified first in peripheral blood leucocytes (12), binding to SDF1 specifically to form the SDF1/CXCR4 axis. The SDF1/CXCR4 axis participates not only in hematopoiesis (13), the immune response (14) and organ development (15), but also in vascular remodeling or neovascularization (16). There are some reports that the SDF1/CXCR4 axis is involved in angiogenesis in some repair processes for tissue damage $(17,18)$. The formation of fissures under the condition of an unusual loading force is a damage process, and the ingrowth of new blood vessels and granulation tissue is considered a repair process; hence, it was speculated that the SDF1/CXCR4 axis may be involved in this pathological activity.

Whether the SDF1/CXCR4 axis plays a role in disc angiogenesis and what role it plays is a key interest. The present study sought to determine the influence of the SDF1/CXCR4 axis on disc angiogenesis by regulating SDF1 expression in nucleus pulposus cells (NPCs) and inhibiting superficial CXCR4 in vascular endothelial cells (VECs) using a molecular compound. This may help create a full understanding of disc degeneration.

\section{Materials and methods}

Cell isolation and culture. Degenerative disc tissues were obtained from the Department of Orthopedics in The First Affiliated Hospital of Chongqing Medical University (from July 2017 to March 2018), and all 10 patients were diagnosed with disc degeneration diseases (lumbar disc herniation, lumbar spinal stenosis or spondylolisthesis). Informed consent was obtained from the donors, and the experimental protocol was approved by the ethics committee of Chongqing Medical University. All specimens was evaluated according to the Pfirrmann classification (19) of preoperative lumbar MRI images, and all specimens were grade III and above (Table I).

Blood was flushed from disc tissues using PBS buffer solution and the nucleus pulposus was carefully separated. Type II collagenase (2\%) was used to digest the nucleus pulposus tissue for $8 \mathrm{~h}$ at $37^{\circ} \mathrm{C}$ the primary NPC suspension was collected. The NPCs were expanded in a monolayer culture with DMEM/F12 (HyClone; GE Healthcare Life Sciences) containing 15\% FBS (CellMax Life). The NPCs were incubated at $37^{\circ} \mathrm{C}$ in a humid atmosphere with $5 \% \mathrm{CO}_{2}$. The medium was changed every 3 days, and the cells were passaged when confluency reached $80 \%$. The passage $1(\mathrm{P} 1)$ cells were chosen for subsequent experiments.

Human umbilical vein endothelial cells (cat. no. hy926; Shanghai Cell Bank, Chinese Academy of Sciences) were cultured with RPMI-1640 (HyClone; GE Healthcare Life Sciences) containing $10 \%$ FBS. The medium wa8s changed every 3 days, and the cells were passaged when confluency reached $80 \%$. To inhibit CXCR4, VECs were exposed to a final concentration of $1 \mu \mathrm{M}$ AMD3100 (Selleck Chemicals) for $1 \mathrm{~h}$ at $37^{\circ} \mathrm{C}$.

\section{Virus transfection of NPCs}

Upregulating SDF1 adenovirus transfection. A total of $10^{5}$ NPCs were plated in six-well plates overnight, then adenovirus-SDF1 (ADV-SDF1; Shanghai GenePharma Co., Ltd.) was added to the wells [multiplicity of infection $(\mathrm{MOI})=100$ ], and adenovirus-negative control (ADV-NC; Shanghai
GenePharma Co., Ltd.) was added in the same way for the control group. The plate was gently shaken and incubated in a $37^{\circ} \mathrm{C}$ incubator; $\sim 12 \mathrm{~h}$ later, the medium was changed and culture of the NPCs continued.

Downregulating SDF1 lentivirus transfection. A total of $10^{5} \mathrm{NPCs}$ were plated in six-well plates overnight, and then, lentivirus-SDF1-RNA interference (RNAi; LV-SDF1-RNAi) [sequence of small interfering (si)RNA: 5'-GTGCATTGACCC GAAGCTAAA-3'; Shanghai GeneChem Co., Ltd.] was added to the wells $(\mathrm{MOI}=10)$ in combination with polybrene at a final concentration of $5 \mu \mathrm{g} / \mathrm{ml}$; lentivirus-negative control (LV-NC; Shanghai GeneChem Co., Ltd.) was added in the same way for the control group. The plate was gently shaken and incubated in a $37^{\circ} \mathrm{C}$ incubator for $6 \mathrm{~h}$, and then the medium was changed. After transfection for 24-48 h, the mRNA expression was determined by reverse transcription quantitative (RT-q)PCR; after transfection for 48-72 h, protein expression was determined by western blotting.

$R T-q P C R$. The NPC medium was discarded, and the NPCs were washed three times using a PBS solution. Total RNA was extracted by TRIzol ${ }^{\circledR}$ (Thermo Fisher Scientific, Inc.). The complementary DNA (cDNA) was reverse transcribed using a RevertAid First Stand cDNA Synthesis kit (Thermo Fisher Scientific, Inc.) following the manufacturer's protocol as follows: $25^{\circ} \mathrm{C}$ for $5 \mathrm{~min}, 42^{\circ} \mathrm{C}$ for $60 \mathrm{~min}$ and $70^{\circ} \mathrm{C}$ for $5 \mathrm{~min}$. The cDNA was subsequently used to perform qPCR with SYBR Select Master Mix (Thermo Fisher Scientific, Inc.) in an ABI-7500 Real-Time PCR system (ABI; Thermo Fisher Scientific, Inc.). The final reaction volume was $10 \mu \mathrm{l}$ and consisted of $1 \mu \mathrm{l} \mathrm{cDNA}, 1 \mu \mathrm{l}$ primer $(5 \mu \mathrm{M} / \mathrm{l}), 3 \mu \mathrm{l}$ nuclease-free water and $5 \mu \mathrm{l} \mathrm{SYBR}$ master mix. SDF1 oligonucleotide primers (forward, 5'-TCAGCCTGAGCTACAGATGCC-3'; reverse, 5'-TCTGAAGGGCACAGTTTGGAG-3'; synthesized by Takara Bio, Inc.) and GAPDH oligonucleotide primers (forward, 5'-CGGAGTCAACGGATTCGGTCGTAT-3'; reverse, 5'-AGCCTTCTCCATGGTGGTGAAGAC-3'; synthesized by Takara Bio, Inc.) were used. The thermal cycling was performed as follows: $50^{\circ} \mathrm{C}$ for $2 \mathrm{~min}, 95^{\circ} \mathrm{C}$ for $2 \mathrm{~min}, 40$ cycles of $95^{\circ} \mathrm{C}$ for $3 \mathrm{sec}$ and $60^{\circ} \mathrm{C}$ for $30 \mathrm{sec}$. The quantification cycle (Cq) value was normalized to GAPDH (20). All samples were analyzed in triplicate.

Western blotting. The lysis of NPCs was performed for total protein with a RIPA lysis buffer (Beyotime Institute of Biotechnology). The concentrations of every group were tested using a BCA protein quantitation kit (Beyotime Institute of Biotechnology). Proteins from cell lysates were separated via $12 \%$ SDS-PAGE (Beyotime Institute of Biotechnology) and $40 \mu \mathrm{g}$ protein was loaded per lane, then transferred to polyvinylidene fluoride membranes (EMD Millipore), which were blocked using $5 \%$ skimmed milk for $1 \mathrm{~h}$ at room temperature. The membranes were incubated at $4^{\circ} \mathrm{C}$ overnight with primary antibodies against SDF1 (1:1,000; ab9797,Abcam) and GAPDH (1:3,000; 10494-1-AP, Proteintech Group, Inc.). After being washed in a TBST solution, the membranes were incubated with horseradish peroxidase-conjugated secondary antibodies (1:1,000; A0208, Beyotime Institute of Biotechnology) for $1 \mathrm{~h}$ at $37^{\circ} \mathrm{C}$. The protein bands were detected using an ECL kit (Wanleibio Co., Ltd.) and a chemiluminescence imaging 
Table I. Specimens data for nucleus pulposus cell isolation.

\begin{tabular}{llclc}
\hline Specimen no. & Sex & Age, years & Segment & $\begin{array}{c}\text { Pfirmann } \\
\text { grade }\end{array}$ \\
\hline 1 & Male & 68 & L4-5 & IV \\
2 & Female & 56 & L4-5 & IV \\
3 & Female & 60 & L4-S1 & V \\
4 & Female & 54 & L5-S1 & III \\
5 & Male & 55 & L4-5 & IV \\
6 & Female & 63 & L5-S1 & V \\
7 & Male & 57 & L4-S1 & IV \\
8 & Female & 60 & L5-S1 & IV \\
9 & Female & 52 & L4-5 & III \\
10 & Male & 60 & L4-5 & V \\
\hline
\end{tabular}

system (Fusion SOLO S, VILBER). Protein expression was quantified using EvolutionCapt SL6 software (v16.0.8.0, Vilber Lourmat Sté).

Immunofluorescence. The coverslips for each condition were taken out of 24-well plates. The NPCs were fixed with $4 \%$ paraformaldehyde for $15 \mathrm{~min}$ and permeabilized with $0.1 \%$ Triton $\mathrm{X}-100$ for $3 \mathrm{~min}$ at room temperature. Then, NPCs were blocked with goat serum (Sigma-Aldrich; Merck KGaA). Subsequently, NPCs were incubated with SDF1 primary antibody (1:200; ab9797, Abcam), type II collagen primary antibody (1:200; ab185430, Abcam) and aggrecan primary antibody $\left(1: 200 ;\right.$ ab36861, Abcam) at $4^{\circ} \mathrm{C}$ overnight. The next day, the slides were washed with PBS solution three times and incubated with FITC or Cy3 conjugated secondary antibodies (A0562 and A0521, Beyotime Institute of Biotechnology)for $1 \mathrm{~h}$ at $37^{\circ} \mathrm{C}$. Cell nuclei were stained with DAPI (Beyotime Institute of Biotechnology) for $3 \mathrm{~min}$ at room temperature. Images were gathered using a fluorescence microscope (magnification, x200; Leica Microsystems, Inc.).

NPC conditioned medium (CM) preparation. According to the literature (21), NPCs from the SDF1 upregulation (Up), degeneration (D) and SDF1 downregulation (Down) groups were prepared and counted. For each group, $5 \times 10^{5}$ cells were seeded in a T25 flask, to which $4 \mathrm{ml}$ DMEM/F12 medium with $10 \%$ FBS was added. These NPCs were incubated at $37^{\circ} \mathrm{C}$ for 3 days. Then, the culture supernatant was obtained and centrifuged at $12,880 \mathrm{x}$ g for $10 \mathrm{mins}$ at $4^{\circ} \mathrm{C}$. The supernatant collected from these NPCs was defined as their CM. The CM of the NPCs was stored at $-80^{\circ} \mathrm{C}$ and used within 2 weeks.

Cell counting kit-8 (CCK-8) assay. A total of 3,000 VECs were seeded in 96 -well plates overnight. The mixed medium was composed of NPC CM and complete medium, at a ratio of 6:4. From each group of NPCs, $100 \mu \mathrm{l}$ mixed medium was added to the relevant wells. After $24 \mathrm{~h}$ of incubation, $10 \mu \mathrm{l}$ CCK-8 solution (Selleck Chemicals) was added to each well. After an additional $1 \mathrm{~h}$ incubation at $37^{\circ} \mathrm{C}$, the absorbance was measured spectrophotometrically at $450 \mathrm{~nm}$. Cell viability was calculated according to the formula: Cell viability $(\%)=\left[\mathrm{A}_{\mathrm{X}}\right.$ group $\left.-A_{\text {blank group }}\right] /\left[\mathrm{A}_{\mathrm{D} \text { group }}-\mathrm{A}_{\text {blank group }}\right] \times 100$.

Transwell cell migration assay. From each group, 5x10 4 NPCs were seeded in 24-well plates and each well was filled with $500 \mu \mathrm{l}$ medium containing $10 \% \mathrm{FBS}$. A total of $10^{4}$ VECs were seeded in a Transwell filter (Corning, Inc.) with $100 \mu \mathrm{l}$ of a medium containing 5\% FBS. The two sets of cells were cultured separately overnight. Filters were placed into the relevant wells the next day, keeping the membrane of the filter immersed in the medium of the lower chamber. After $12 \mathrm{~h}$ of co-culture, the upper filters were removed and the cells of the upper membrane surface were removed with a cotton swab. The filters were fixed in $4 \%$ paraformaldehyde solution for 15 mins at room temperature. After washing with PBS solution three times, the filters were immersed in $0.1 \%$ crystal violet solution for 10 mins at room temperature. The excess crystal violet was rinsed with PBS solution. The images were captured using an inverted light microscope (Leica Microsystems $\mathrm{GmbH}$ ). A total of five random fields of view in each group were acquired and analyzed using ImageJ software (v1.51; National Institutes of Health).

Tube formation assay. The serum starved VECs were prepared one night in advance. Matrigel (BD Biosciences) was melted on ice, and $50 \mu \mathrm{l}$ of Matrigel was placed into the wells of a 96-well plate, ensuring that the Matrigel was homogeneous. The Matrigel was polymerized at $37^{\circ} \mathrm{C}$ for $30 \mathrm{~min}$. The VECs were acquired and resuspended using a mixed medium (NPC CM:complete medium with $10 \% \mathrm{FBS}$ at a ratio of 6:4). A total of $2 \times 10^{4} \mathrm{VECs}$ in $100 \mu \mathrm{l}$ mixed medium were placed onto the Matrigel coated wells and incubated at $37^{\circ} \mathrm{C}$ for another $4 \mathrm{~h}$. The tube structure was formed and images were captured using an inverted light microscope (Leica Microsystems $\mathrm{GmbH}$ ). A total of five random fields of view in each group were acquired and analyzed using ImageJ software (v1.51, National Institutes of Health).

Statistical analysis. All data are presented as the mean \pm SD. All experiments were repeated three times. SPSS 19.0 (IBM Corp.) was used for the statistical analysis. Single variable multigroup data were analyzed by a one-way ANOVA; two variable multigroup data were analyzed by a two-way ANOVA and a Tukey's post hoc test. $\mathrm{P}<0.05$ was considered to indicate a statistically significant difference.

\section{Results}

Culture and identification of primary NPCs. The primary NPCs were obtained and monolayer cultured in vitro. Firstly, the phenotype of the NPCs was identified by cell immunofluorescence of type II collagen and aggrecan (22). The acquired fluorescent images showed that the cells were polygonal and simultaneously appeared as having green fluorescence (aggrecan) and red fluorescence (type II collagen), still showing a chondrocyte-like phenotype, and were identified as NPCs (Fig. 1). The P1 generation cells were used for subsequent experiments to keep the phenotype to the greatest extent.

Upregulation and downregulation of SDF1 expression in NPCs. To explore the effect of the different expression of SDF1 in NPCs on VECs, it was first necessary to obtain 

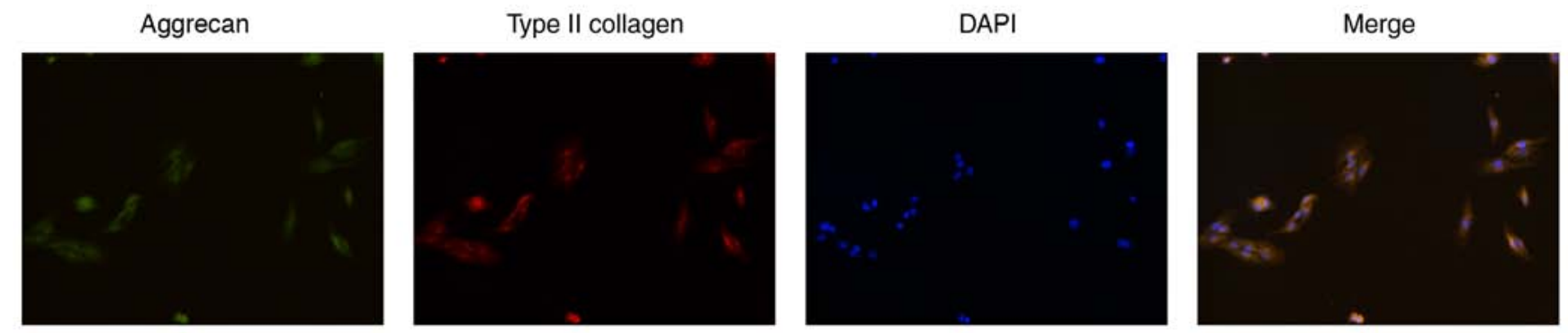

Figure 1. Identification of primary NPCs. NPCs were identified by cell immunofluorescence of type II collagen and aggrecan. The cells simultaneously expressed aggrecan (green fluorescence) and type II collagen (red fluorescence) while still showing a chondrocyte-like phenotype, and were identified as NPCs. Magnification, x200. NPCs, nucleus pulposus cells.

NPCs with different expression levels of SDF1. The expression of SDF1 in NPCs was regulated by virus transfection and identified using RT-qPCR, western blotting and cellular immunofluorescence technology. The NPCs not treated with the virus were defined as the D group. After transfection with the adenovirus carrying the SDF1 sequence, total RNA and protein were obtained. The PCR results showed that the relative mRNA expression of SDF1 in the Up group was significantly upregulated compared with the $\mathrm{D}$ and negative control (NC) groups (Fig. 2A), and the western blotting results showed that the expression of SDF1 in the Up group was significantly upregulated compared with the D and NC groups (Fig. 2C and D). The cells of the adenovirus transfected group were defined as the Up group. After transfection with a lentivirus carrying siRNA sequences, total RNA and protein were obtained. The qPCR results showed that the relative mRNA expression of SDF1 in the Down group was significantly downregulated compared with the D and NC groups (Fig. 2B), and the western blotting results showed that the expression of SDF1 in the Down group was significantly downregulated compared with the $\mathrm{D}$ and NC groups (Fig. 2E and F). The cells of the lentivirus transfection group were defined as the Down group. Later, using cellular immunofluorescence technology, the expression of SDF1 (green) in the three groups of NPCs could be visualized (Fig. 3). The Up group showed the strongest green fluorescence, the D group showed medium green fluorescence, and the Down group showed the weakest green fluorescence. Finally, groups of NPCs with different expression levels of SDF1 were successfully obtained for subsequent experiments.

Effect of NPCs on VECs in a co-culture system. To detect the effect of NPCs (or the CM of NPCs) with different expression levels of SDF1 on VECs, a co-culture system was employed. Later, VECs were pre-incubated with AMD3100 to detect the effect of CXCR4 on the angiogenesis of VECs. The cell viability of VECs among the three groups was significantly enhanced as the expression of SDF1 increased; after pre-incubation with AMD3100, the viability of VECs in the D and Up groups was significantly decreased (Fig. 4). The migration ability was monitored by counting the number of transmembrane VECs, and the analysis was conducted using ImageJ software. As the expression of SDF1 increased, the number of transmembrane VECs significantly increased among the three groups; after preincubation with AMD3100, the number of transmembrane VECs in the D and Up groups significantly decreased (Fig. 5).
The tube formation ability of VECs was monitored by measuring the total branching length, and measurements were conducted using ImageJ software. As the expression of SDF1 increased, the total branching length significantly increased among the three groups; after pre-incubation with AMD3100, the total branching length in the D and Up groups significantly decreased (Fig. 6). It was also noted that the cell viability, migration and tube formation ability of VECs in the Down group decreased before and after incubation with AMD3100, but there was no significant difference.

\section{Discussion}

Chemokines are a class of polypeptides consisting of $\sim 100$ amino acids that act as cytokines leading to chemotaxis, and SDF1 is an important example of these proteins. Many types of cells express SDF1, such as cardiomyocytes, endothelial cells, smooth muscle cells, macrophages and progenitor cells $(23,24)$, and it is involved in an array of physiological and pathological activities in vivo. In the field of orthopedics, a previous report demonstrated that the SDF1/CXCR4 axis participated in the regulation of changes in matrix components and cartilage degeneration during osteoarthritis (25). Intervertebral discs also belong to the motor system, the same as articular cartilage, enduring high stress and the lack of a blood supply. Degenerative disc disease and osteoarthritis are both degenerative diseases of the motor system. Therefore, it was speculated that the SDF1/CXCR4 axis may participate in the disc degeneration process.

Zhang et al (26) reported that SDF1 and CXCR4 expression were upregulated in degenerated discs, as detected by immunohistochemistry. Further research from Liu et al (27) revealed that SDF1/CXCR4 was not only increased in degenerated intervertebral discs, but also resulted in disc degeneration by inducing apoptosis in NPCs. SDF1 is a secretory protein and is secreted into the extracellular matrix after its production by cells. Previous reports $(26,27)$ used immunohistochemical methods to illustrate the expression of SDF1, and showed that there are large areas of positive staining in the extracellular matrix of degenerated discs, but whether SDF1 is expressed by NPCs was not clear. In the present experiments, the NPCs of degenerated intervertebral discs were identified as the source of SDF1 using RT-qPCR, western blotting and immunofluorescence analysis. It was therefore concluded that the degenerated NPCs can secret SDF1 to play a physiological and pathological role. 
A

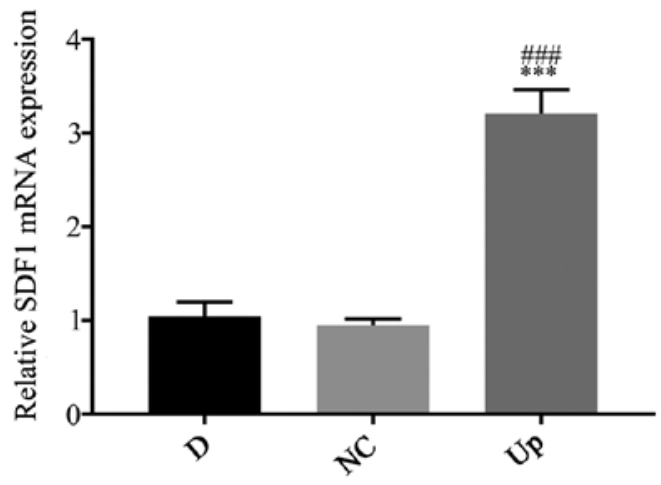

$\mathrm{C}$

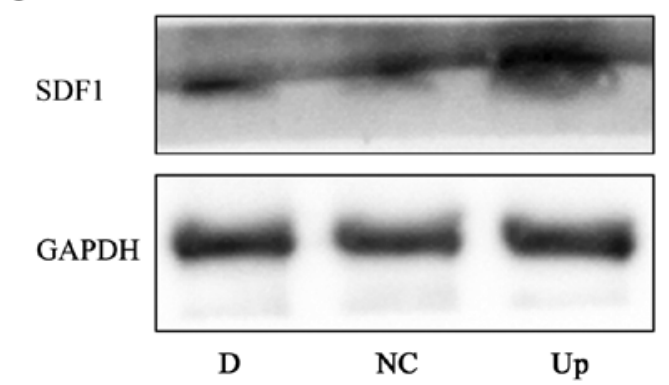

E

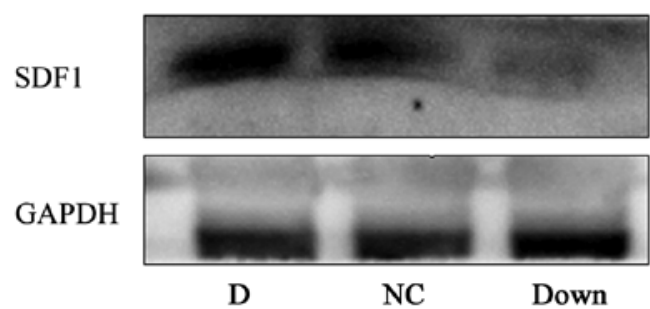

$B$

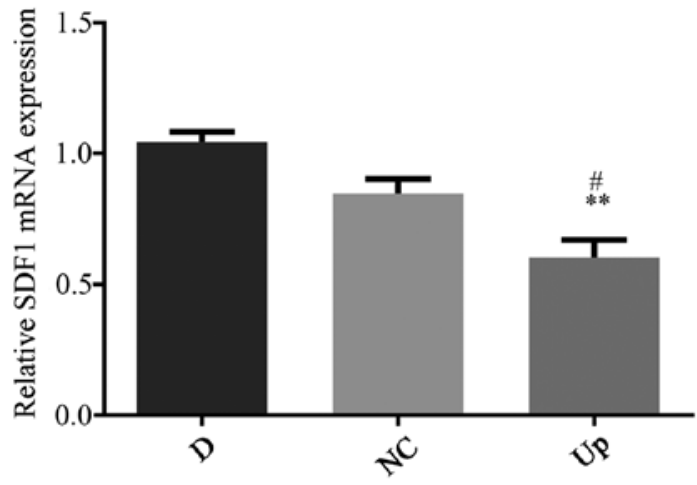

$\mathrm{D}$
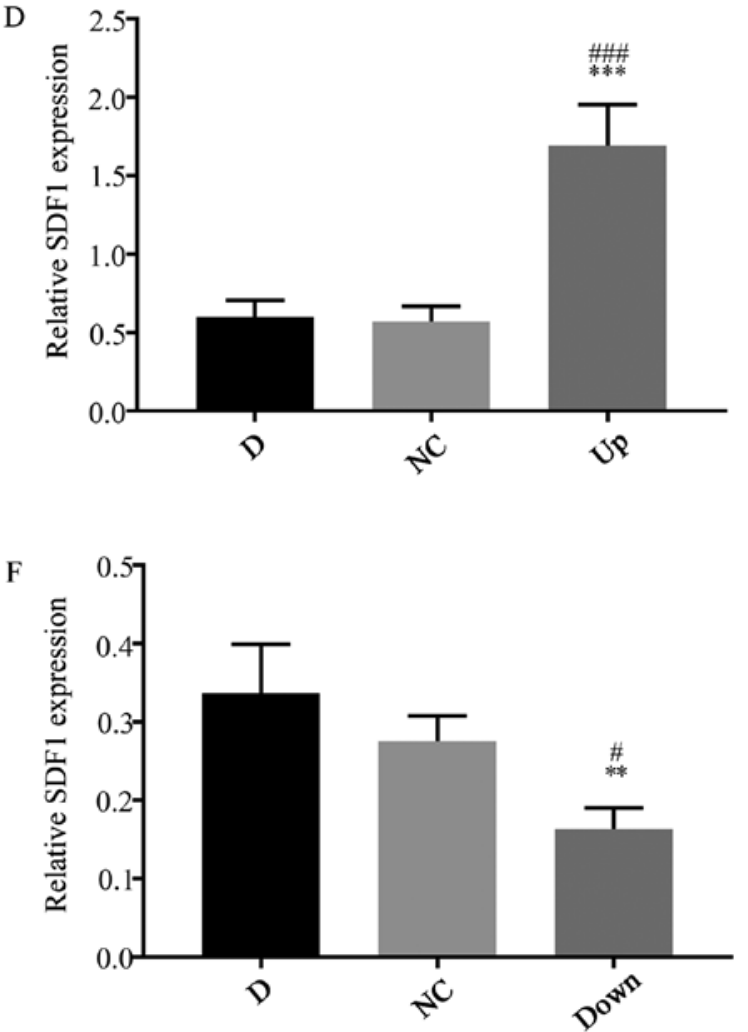

Figure 2. Upregulation and downregulation of SDF1 expression in nucleus pulposus cells. (A) RT-qPCR showing that the mRNA expression level of SDF1 in the Up group was significantly upregulated compared with the D and NC groups. (B) RT-qPCR showing that the mRNA expression level of SDF1 in the Down group was significantly downregulated compared with the D and NC groups. (C) Western blot analysis showing the SDF1 expression level in the D, NC and Up groups. (D) The statistical results of (C), showing that SDF1 expression in the Up group was significantly increased compared with the D and NC groups. (E) Western blot analysis showing the SDF1 expression levels in the D, NC and Down groups. (F) The statistical results of (E), showing that $\mathrm{SDF} 1$ expression in the Down group was significantly decreased compared with the $\mathrm{D}$ and $\mathrm{NC}$ groups. ${ }^{* *} \mathrm{P}<0.01,{ }^{* * * *} \mathrm{P}<0.001$ vs. respective $\mathrm{D}$ group. ${ }^{*} \mathrm{P}<0.05$,

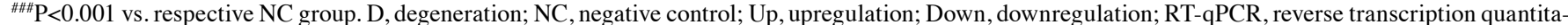
tive PCR; SDF1, stromal cell derived factor 1.

In this study, the expression of SDF1 in NPCs was upregulated and downregulated by virus transfection, and the expression was successfully confirmed at the mRNA and protein level. Then, using a conditioned medium produced by NPCs or by direct co-culture, the interaction between NPCs and VECs was observed. The results showed that the proliferation, migration and tube formation of VECs were enhanced with the increase in SDF1 expression. The intervertebral disc is an organ that lacks blood vessels, and only the one-third of the outer layer of the annulus has blood vessels; this is a result of the balance between anti-vascular ingrowth factors and vascular ingrowth factors. Under normal conditions, the aggrecan component contained in the nucleus pulposus has an inhibitory effect on vascular ingrowth (28). In the case of discogenic LBP, it can be seen that the new blood vessels grow from the annulus fibrosus into the nucleus pulposus, which is accompanied by the occurrence of nucleus pulposus fibrosis (29). This indicates that when intervertebral disc degeneration occurs, the aggrecan component in the nucleus pulposus is reduced, which works as a factor of anti-vascular ingrowth. To the contrary, the factors that promote vascular ingrowth are increased. The comprehensive result is that neovascularization occurs into the degenerative intervertebral disc, even deep into the nucleus pulposus, causing painful intervertebral disc degeneration. As a factor of angiogenesis, SDF1 has been reported in many physiological and pathological 

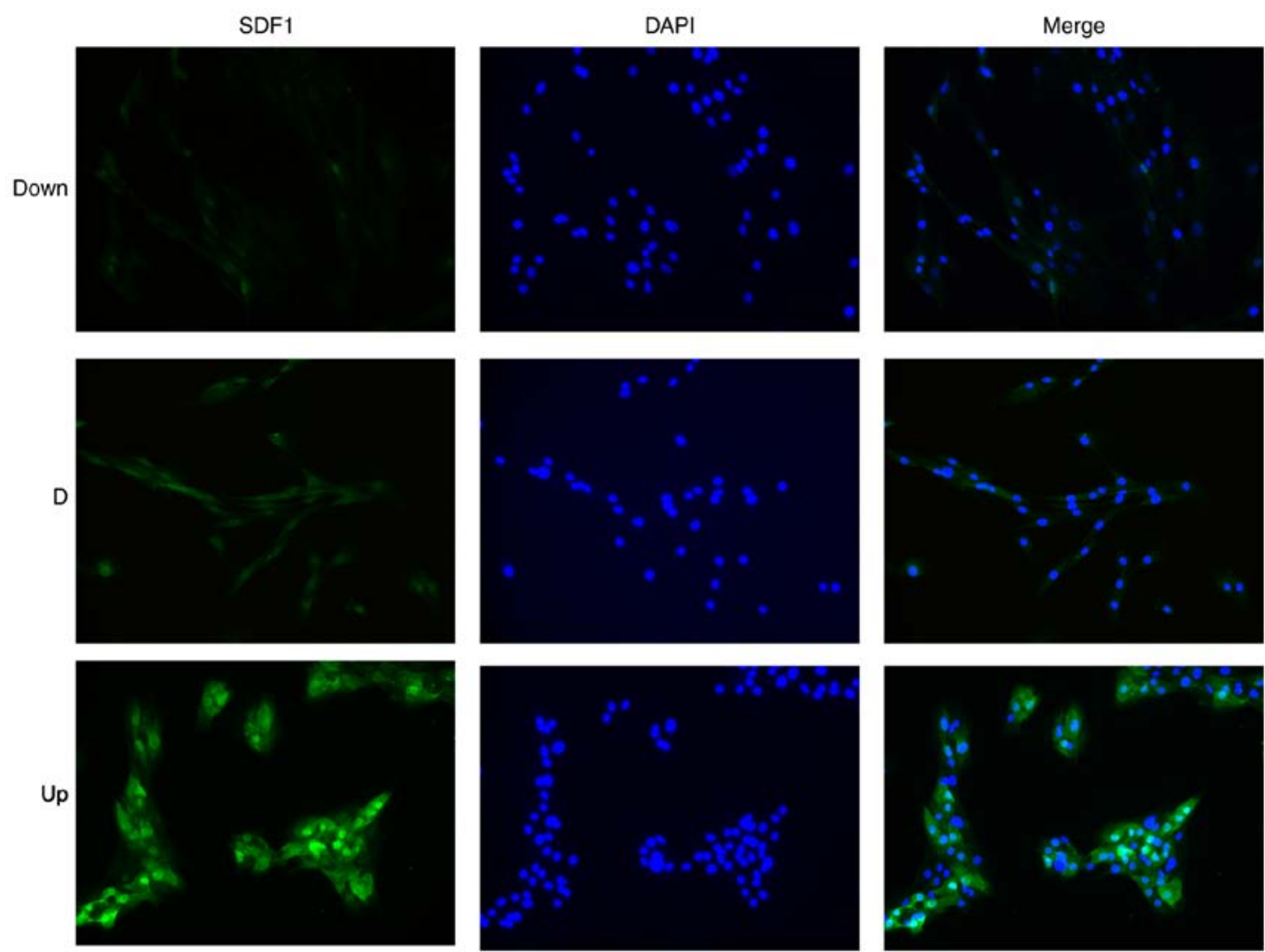

Figure 3. SDF1 immunofluorescence of nucleus pulposus cells in the Down, D and Up groups. The fluorescence intensity in the Down group was the weakest, and the fluorescence intensity in the Up group was the strongest. Magnification, x200. Down, downregulation; D, degeneration; Up, upregulation; SDF1, stromal cell derived factor 1 .

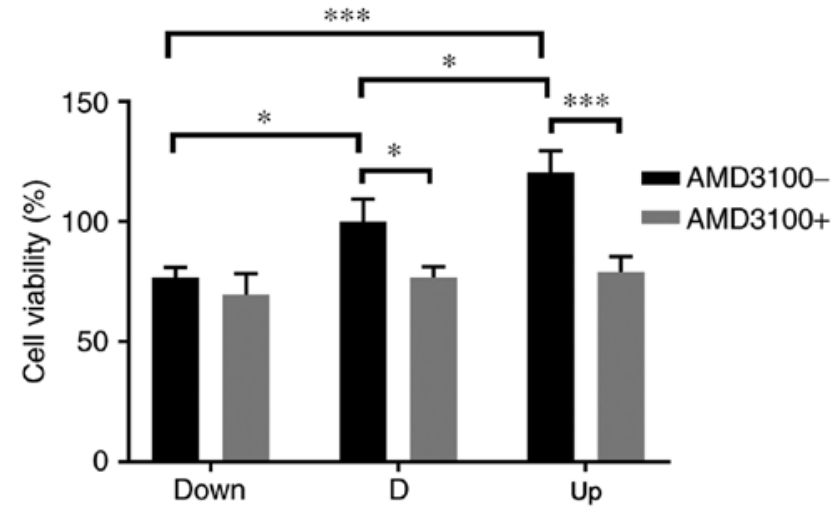

Figure 4. Influence of nucleus pulposus cells on the cell viability of VECs in the co-culture system. Before adding AMD3100, the cell viability of VECs increased as stromal cell derived factor 1 increased in the Down, D and Up groups, and there were significant differences among the groups. After adding AMD3100, the cell viability in the D and Up groups decreased significantly. There was no significant difference in the Down group before or after adding AMD3100. ${ }^{*} \mathrm{P}<0.05,{ }^{* * *} \mathrm{P}<0.001$. VECs, vascular endothelial cells; Down, downregulation; D, degeneration; Up, upregulation; AMD3100without adding AMD3100 into the co-culture system; AMD3100+, adding AMD3100 into the co-culture system.

processes. Cai et al (30) reported that SDF1, which is enhanced during hyperglycemia, can promote choroidal vascularization. Yu et al (31) reported that SDF1, which was highly expressed in renal cells, promoted the neovascularization of tumors. Virgintino et al (32) reported that SDF1 was involved in the regulation of angiogenesis during human brain development.
The present results showed that with the increase in SDF1 expression in NPCs, the proliferation and chemotactic migration ability of VECs were enhanced. The ability of tube-like structure formation was also enhanced, which is involved in the synthesis of a series of proteins, collagen and proteases in VECs (33). These results suggested that SDF1 also plays an important role in the process of vascularization in degenerated intervertebral discs. Furthermore, the degree of vascularization in the intervertebral disc is positively correlated with the degree of degeneration (10).

CXCR4 is a specific receptor for SDF1 and is expressed in a variety of cells, including VECs. SDF1 and CXCR4 constitute a signaling axis and participate in the pathological process of various diseases, such as HIV infection, tumor metastasis, pathological angiogenesis, myocardial ischemia and pulmonary fibrosis (34). CXCR4 not only promotes angiogenesis in normal tissues, such as the cornea and retina, but also promotes angiogenesis under pathological conditions such as tumors. In this experiment, the interaction of each group of NPCs (or the CM of NPCs) and VECs exhibited properties of facilitating angiogenesis. However, after pre-incubation of the VECs with AMD3100, which is a small molecule inhibitor of CXCR4, the effects of each group of NPCs (or the CM of NPCs), not only on the endothelial cell proliferation and chemotactic migration but also on the tube-like structure formation, were blocked and declined to varying degrees. These results suggested that SDF1 passes signals through CXCR4 on the endothelial cell surface, and the SDF1/CXCR4 axis plays an important role in promoting the angiogenesis of VECs induced by NPCs. At the 
A

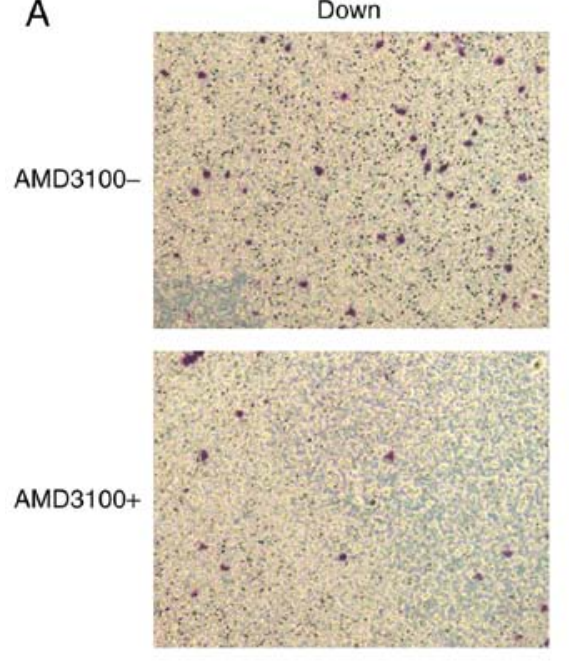

D
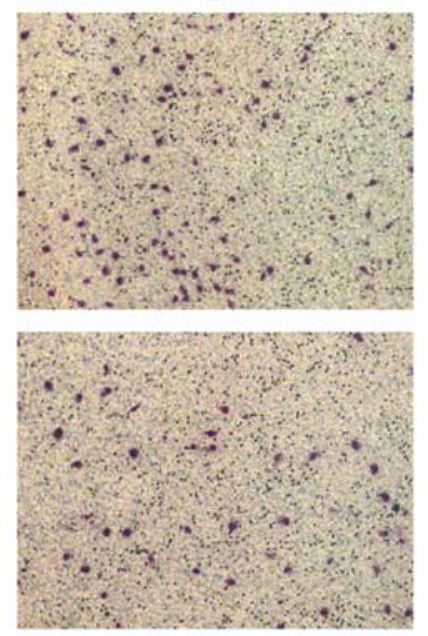

Up
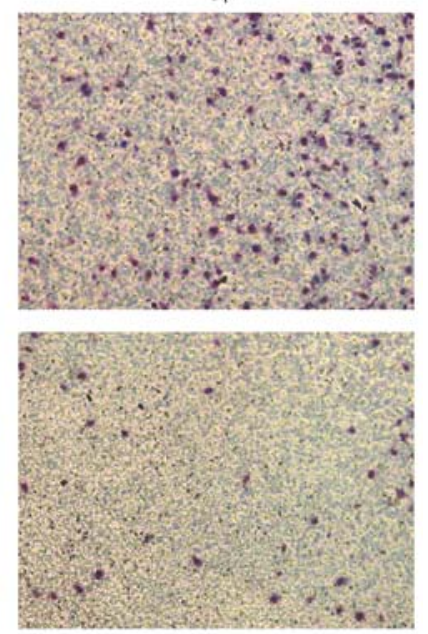

B

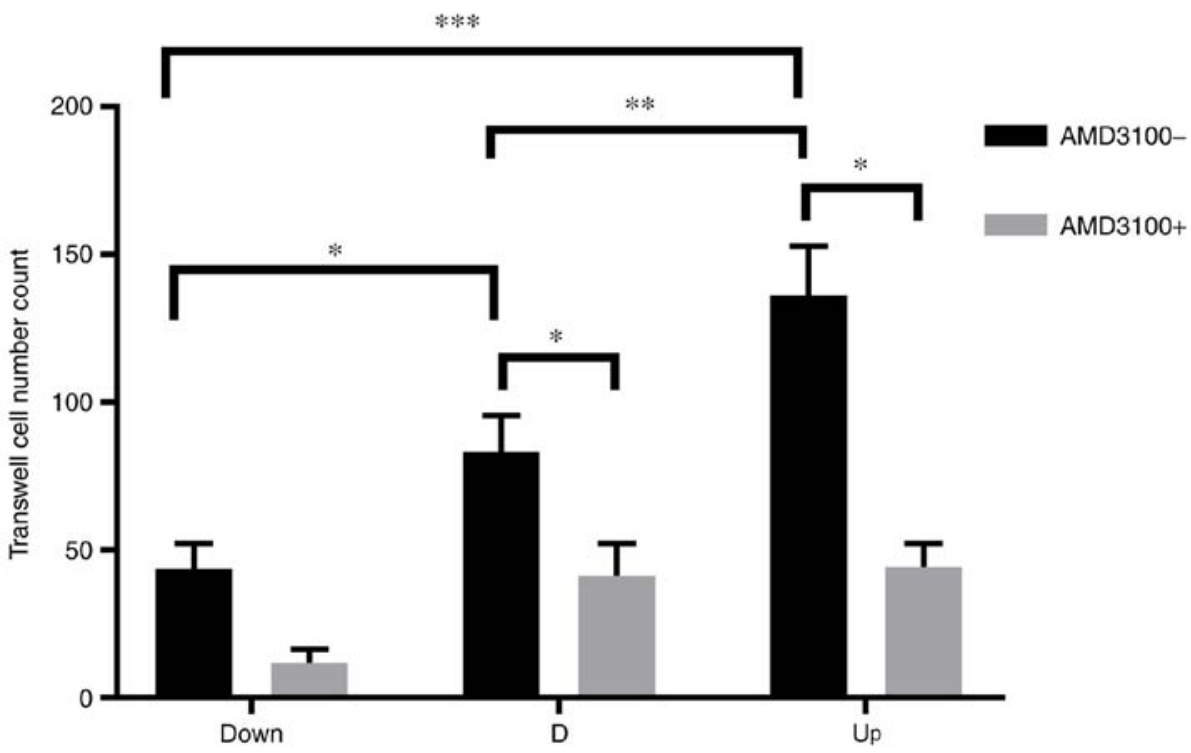

Figure 5. Influence of nucleus pulposus cells on the migration of VECs in the co-culture system. (A) In the co-culture system, before adding AMD3100, the number of transmembrane VECs increased as stromal cell derived factor increased in the Down, D and Up groups. After adding AMD3100, the number of transmembrane VECs decreased in all three groups. Magnification, x200. (B) Statistical results, showing that there was a significant difference in the number of transmembrane VECs between each group before adding AMD3100. After adding AMD3100, the number of transmembrane VECs in the D and Up groups decreased significantly. There was no significant difference in the Down group before or after adding AMD3100. ${ }^{*} \mathrm{P}<0.05,{ }^{* *} \mathrm{P}<0.01,{ }^{* * *} \mathrm{P}<0.001$. VECs, vascular endothelial cells; Down, downregulation; D, degeneration; Up, upregulation; AMD3100-, without adding AMD3100 into the co-culture system; AMD3100+, adding AMD3100 into the co-culture system.

same time, according to a previous study (35), it can be speculated that in degenerated intervertebral discs, because of the large amount of inflammatory substances such as IL-1 $\beta$ (36), the expression of CXCR4 in VECs will be further upregulated, and the response to SDF1 will be more intense, showing stronger chemotaxis. The macroscopic manifestation is that in degenerated intervertebral discs accompanied by a large number of inflammatory mediators, more neovascularization will lead to ingrowth into the nucleus pulposus.

The present results also showed that in the SDF1 downregulation group (Down), there was a further decrease in the cell viability, migration ability and tube formation ability before and after incubation with AMD3100, but there were no significant differences. It was considered that the decreased expression of SDF1 greatly affected the angiogenesis of VECs. Therefore, further inhibition of CXCR4 did not further inhibit angiogenesis. On the other hand, these results also indicated that the effect of SDF1 on angiogenesis may be as important as that of CXCR4.

The shortcoming of these experiments is that the NPCs and VECs were in a monolayer culture with a sufficient oxygen supply. Although the P1 generation of NPCs were chosen for this experiment to keep the cell phenotype as close to the original generation as possible, there were still differences in regard to the high pressure, low oxygen and acidic environment observed in vivo. Therefore, the addition of continuous pressure (or a regularly changing pressure to simulate the change of pressure in vivo) under hypoxic culture conditions may be closer to the in vivo situation, and will provide more in-depth and objective data in exploring the angiogenesis of degenerated intervertebral discs.

The present study upregulated and downregulated the expression of SDF1 in degenerated NPCs by virus transfection and identified the main source of SDF1 in nucleus pulposus 
A
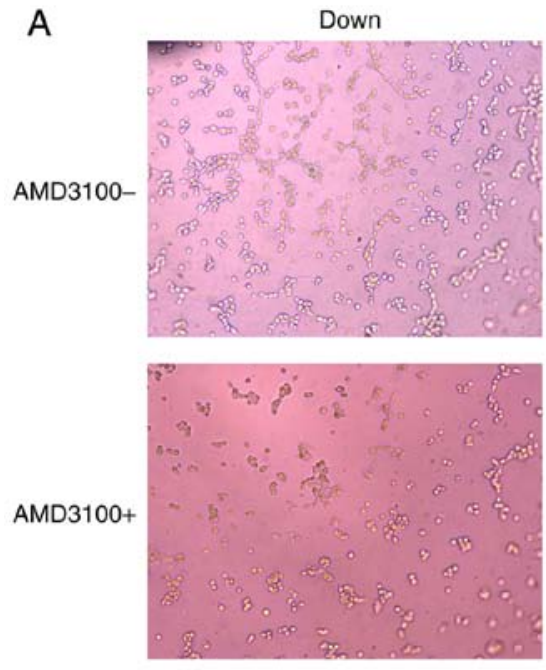

D
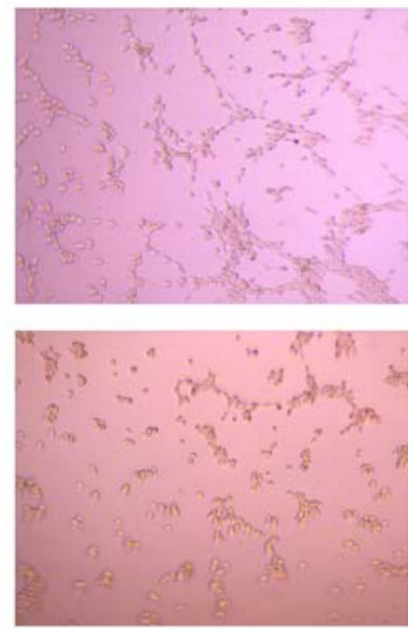

Up
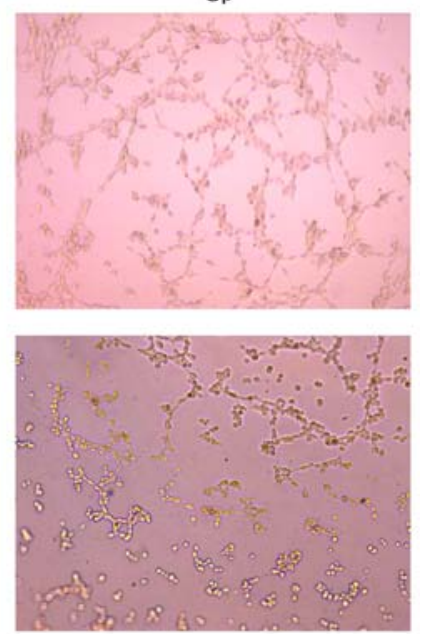

B

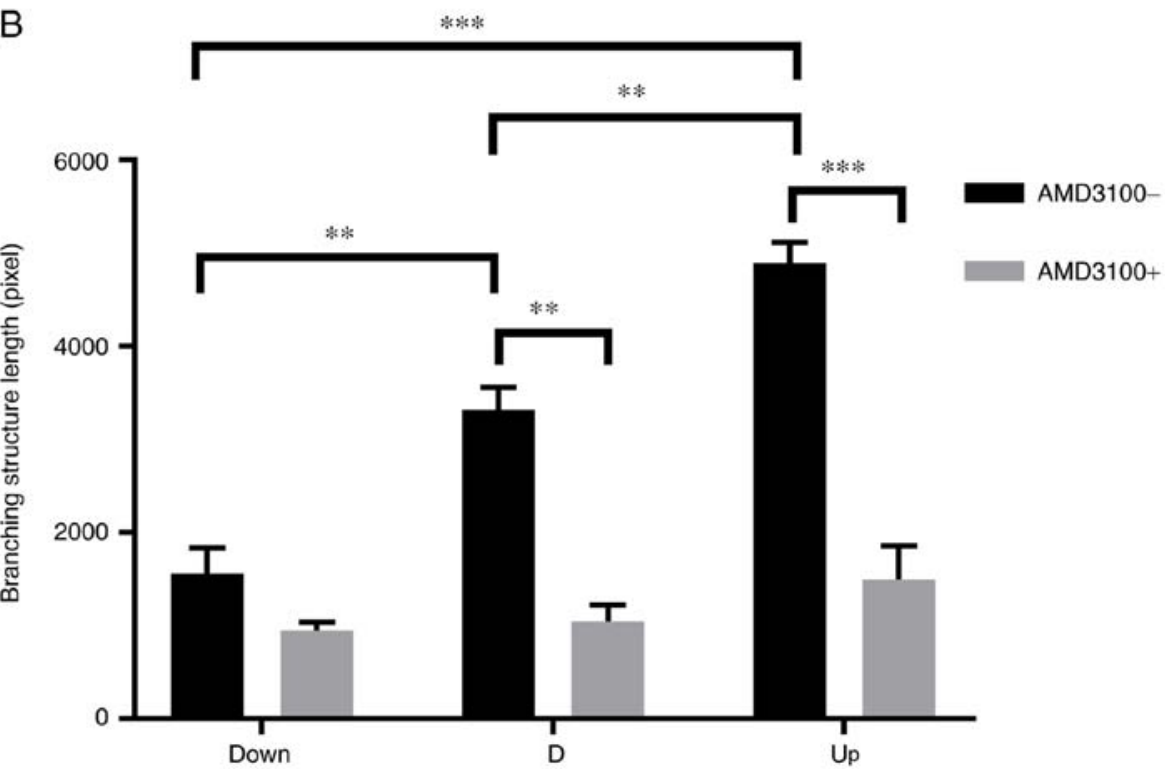

Figure 6. Influence of nucleus pulposus cells on the tube formation of vascular endothelial cells in the co-culture system. (A) In the co-culture system, before adding AMD3100, the branching structures were increased as stromal cell derived factor 1 increased in the Down, D and Up groups. After adding AMD3100, the branching structures were decreased in all three groups. Magnification, x200. (B) Statistical results, showing that there was a branching structure length difference between each group before adding AMD3100. After adding AMD3100, the branching structure length in the D and Up groups decreased significantly. There was no significant difference in the Down group before or after adding AMD3100. ${ }^{* *} \mathrm{P}<0.01,{ }^{* * * *} \mathrm{P}<0.001$. Down, downregulation; $\mathrm{D}$, degeneration; Up, upregulation; AMD3100-, without adding AMD3100 into the co-culture system; AMD3100+, adding AMD3100 into the co-culture system.

tissue. At the same time, the NPCs (or CM of NPCs) with different SDF1 expression levels were co-cultured with VECs, and the results suggested that the SDF1/CXCR4 axis plays a role in new blood vessel ingrowth into degenerated intervertebral discs. As the expression of SDF1/CXCR4 increases, the effect on angiogenesis is also increased. This will help to further understand the process of intervertebral disc degeneration, and provide new ideas for exploring the pathogenesis and treatment of disc degeneration.

\section{Acknowledgements}

The authors thank the Department of Orthopedics at The First Affiliated Hospital of Chongqing Medical University for their collaboration and support. The authors express special gratitude to the Chongqing Key Laboratory of Ophthalmology for providing the experimental platform.

\section{Funding}

The present study was supported by the Guizhou Science and Technology Department Cooperation Project (2015) (grant no. 2152).

\section{Availability of data and materials}

The datasets used and/or analyzed during the current study are included in this published article.

\section{Authors' contributions}

HZ participated in the study design, data collection, statistical analysis, manuscript preparation and literature search. BH participated in the study design, data collection, statistical analysis and collection of funds. 


\section{Ethics approval and consent to participate}

Informed consent was obtained from all donors, and the experimental protocol was approved by the Ethics Committee of Chongqing Medical University.

\section{Patient consent for publication}

Not applicable.

\section{Competing interests}

The authors declare that they have no competing interests.

\section{References}

1. Andersson GB: Epidemiological features of chronic low-back pain. Lancet 354: 581-585, 1999.

2. Martin BI, Turner JA, Mirza SK, Lee MJ, Comstock BA and Deyo RA: Trends in health care expenditures, utilization, and health status among US adults with spine problems, 1997-2006. Spine (Phila Pa 1976) 34: 2077-2084, 2009.

3. Dieleman JL, Baral R, Birger M, Bui AL, Bulchis A, Chapin A, Hamavid H, Horst C, Johnson EK, Joseph J, et al: US spending on personal health care and public health, 1996-2013. JAMA 316 2627-2646, 2016.

4. O'Halloran DM and Pandit AS: Tissue-engineering approach to regenerating the intervertebral disc. Tissue Eng 13: 1927-1954, 2007.

5. Urban JP, Smith S and Fairbank JC: Nutrition of the intervertebral disc. Spine (Phila Pa 1976) 29: 2700-2709, 2004.

6. Matveeva N, Zivadinovik J, Zdravkovska M, Jovevska S and Bojadzieva B: Histological composition of lumbar disc herniations related to the type of herniation and to the age. Bratisl Lek Listy 113: 712-717, 2012.

7. Ahmed M, Bjurholm A, Kreicbergs A and Schuftzberg A Neuropeptide $Y$, tyrosine hydroxylase and vasoactive intestina polypeptide-immunoreactive nerve fibers in the vertebral bodies, discs, dura mater and spinal ligaments of the rat lumbar spine. Spine (Phila Pa 1976) 18: 268-273, 1993.

8. Stefanakis M, Al-Abbasi M, Harding I, Pollintine P, Dolan P, Tarlton J and Adams MA: Annulus fissures are mechanically and chemically conducive to the ingrowth of nerves and blood vessels. Spine (Phila Pa 1976) 37: 1883-1891, 2012.

9. Freemont AJ, Watkins A, Le Maitre C, Baird P, Jeziorska M, Knight MT, Ross ER, O'Brien JP and Hoyland JA: Nerve growth factor expression and innervation of the painful intervertebral disc. J Pathol 197: 286-292, 2002.

10. Rätsep T, Minajeva A and Asser T: Relationship between neovascularization and degenerative changes in herniated lumbar intervertebral discs. Eur Spine J 22: 2474-2480, 2013.

11. Nagasawa T, Kikutani $\mathrm{H}$ and Kishimoto T: Molecular cloning and structure of a pre-B-cell growth-stimulating factor. Proc Natl Acad Sci USA 91: 2305-2309, 1994.

12. Loetscher M, Geiser T, O'Reilly T, Zwahlen R, Baggiolini M and Moser B: Cloning of a human seven-transmembrane domain receptor, LESTR, that is highly expressed in leukocytes. J Bio Chem 269: 232-237, 1994.

13. Zou YR, Kottmann AH, Kuroda M, Taniuchi I and Littman DR: Function of the chemokine receptor CXCR4 in haematopoiesis and in cerebellar development. Nature 393: 595-599, 1998.

14. Moser B and Loetscher P: Lymphocyte traffic control by chemokines. Nat Immunol 2: 123-128, 2001.

15. Katsumoto $\mathrm{K}$ and Kume $\mathrm{S}$ : The role of CXCL12-CXCR4 signaling pathway in pancreatic development. Theranostics 3 : 11-17, 2013.

16. Sainz J and Sata M: CXCR4, a key modulator of vascular progenitor cells. Arterioscler Thromb Vasc Biol 27: 263-265, 2007.

17. Sasaki T, Fukazawa R, Ogawa S, Kanno S, Nitta T, Ochi M and Shimizu K: Stromal cell-derived factor-1alpha improves infarcted heart function through angiogenesis in mice. Pediatr Int 49: 966-971, 2007
18. Shyu WC, Lin SZ, Yen PS, Su CY, Chen DC, Wang HJ and Li H: Stromal cell-derived factor-1 alpha promotes neuroprotection, angiogenesis, and mobilization/homing of bone marrow-derived cells in stroke rats. J Pharmacol Exp Ther 324: 834-849, 2008.

19. Pfirrmann CW, Metzdorf A, Zanetti M, Hodler J and Boos N: Magnetic resonance classification of lumbar intervertebral disc degeneration. Spine (Phila Pa 1976) 26: 1873-1878, 2001.

20. Livak KJ and Schmittgen TD: Analysis of relative gene expression data using real-time quantitative PCR and the 2(-Delta Delta C(T)) method. Methods 25: 402-408, 2001.

21. Kwon WK, Moon HJ, Kwon TH, Park YK and Kim JH: Influence of rabbit notochordal cells on symptomatic intervertebral disc degeneration: Anti-angiogenic capacity on human endothelial cell proliferation under hypoxia. Osteoarthritis Cartilage 25: 1738-1746, 2017.

22. Vernon-Roberts B, Moore RJ and Fraser RD: The natural history of age-related disc degeneration: The pathology and sequelae of tears. Spine (Phila Pa 1976) 32: 2797-2804, 2007.

23. Jaerve A, Schira J and Müller HW: Concise review: The potential of stromal cell-derived factor 1 and its receptors to promote stem cell functions in spinal cord repair. Stem Cells Transl Med 1: 732-739, 2012.

24. Dong F, Harvey J, Finan A, Weber K, Agarwal U and Penn MS: Myocardial CXCR4 expression is required for mesenchymal stem cell mediated repair following acute myocardial infarction. Circulation 126: 314-324, 2012.

25. Lu W, Shi J, Zhang J, Lv Z, Guo F, Huang H, Zhu W and Chen A: CXCL12/CXCR4 axis regulates aggrecanase activation and cartilage degradation in a post-traumatic osteoarthritis rat model. Int J Mol Sci 17: pii: E1522, 2016.

26. Zhang H, Zhang L, Chen L, Li W, Li F and Chen Q: Stromal cell-derived factor-1 and its receptor CXCR4 are upregulated expression in degenerated intervertebral discs. Int J Med Sci 11: 240-245, 2014

27. Liu Z, Ma C, Shen J, Wang D, Hao J and Hu Z: SDF-1/CXCR4 axis induces apoptosis of human degenerative nucleus pulposus cells via the NF-кB pathway. Mol Med Rep 14: 783-789, 2016.

28. Johnson WE, Caterson B, Eisenstein SM and Roberts S: Human intervertebral disc aggrecan inhibits endothelial cell adhesion and cell migration in vitro. Spine (Phila Pa 1976) 30: 1139-1147, 2005.

29. Peng B, Chen J, Kuang Z, Li D, Pang X and Zhang X: Expression and role of connective tissue growth factor in painful disc fibrosis and degeneration. Spine (Phila Pa 1976) 34: E178-E182, 2009.

30. Cai Y, Li X, Wang YS, Shi YY, Ye Z, Yang GD, Dou GR, Hou HY, Yang N, Cao XR and Lu ZF: Hyperglycemia promotes vasculogenesis in choroidal neovascularization in diabetic mice by stimulating VEGF and SDF-1 expression in retinal pigment epithelial cells. Exp Eye Res 123: 87-96, 2014.

31. Yu P, Ge YZ, Zhao Y, Wu JP, Wu R, Zhou LH and Jia RP. Identification and significance of mobilized endothelial progenitor cells in tumor neovascularization of renal cell carcinoma. Tumour Biol 35: 9331-9341, 2014.

32. Virgintino D, Errede M, Rizzi M, Girolamo F, Strippoli M, Wälchli T, Robertson D, Frei K and Roncali L: The CXCL12/CXCR4/CXCR7 ligand-receptor system regulates neuro-glio-vascular interactions and vessel growth during human brain development. J Inherit Metab Dis 36: 455-566, 2013.

33. Arnaoutova I and Kleinman HK: In vitro angiogenesis: Endothelial cell tube formation on gelled basement membrane extract. Nat Protoc 5: 628-635, 2010.

34. Debnath B, Xu SL, Grande F, Garofalo A and Neamati N: Small molecule inhibitors of CXCR4. Theranostics 3: 47-75, 2013.

35. Gupta SK, Lysko PG, Pillarisetti K, Ohlstein E and Stadel JM: Chemokine receptors in human endothelial cells. Functional expression of CXCR4 and its transcriptional regulation by inflammatory cytokines. J Biol Chem 273: 4282-4287, 1998.

36. Yang W, Yu XH, Wang C, He WS, Zhang SJ, Yan YG, Zhang J, Xiang YX and Wang WJ: Interleukin-1 $\beta$ in intervertebral disk degeneration. Clin Chim Acta 450: 262-272, 2015.

This work is licensed under a Creative Commons Attribution-NonCommercial-NoDerivatives 4.0 International (CC BY-NC-ND 4.0) License. 